\title{
MOLECULAR CLOUDS IN DWARF IRREGULAR GALAXIES
}

\author{
C. Henkel
}

Max-Planck-Institut für Radioastronomie, Auf dem Hügel 69, 5300 Bonn 1, F.R.G.

The study of molecular clouds in dwarf galaxies was, until recently, an arduous task and provided detections in only a handful of sources (see Thronson and Bally 1986; Wiklind et al. 1986; Tacconi and Young 1987; Arnoult et al. 1988). Line strengths observed in CO were small $(<100 \mathrm{mK})$ and measurements were confined to one position per source, thus inhibiting any detailed work. However, dwarf galaxies appear to be much more abundant than either spirals or large ellipticals and play an important role in our understanding of star and galaxy formation (see e.g. Gerola et al. 1980; Gallagher and Hunter 1984; Silk et al. 1987). With small rotational velocities (typically $50-100 \mathrm{kms}^{-1}$ ), the absence of density waves, and low metallicities, they provide, by "local" standards, an interstellar environment with even more extreme conditions than the outer Galaxy. Detailed molecular studies are hence important.

In small galaxies, star formation is believed to occur in bursts which are followed by long inactive periods during which new molecular material may be built up (e.g. Gerola et al. 1980). The observation of blue compact galaxies proves that star formation can proceed, during the burst phase, as prodiguously as in spirals and a detailed investigation of molecular clouds in this as in the quiescent phase would be worthwhile. What are the properties of the individual clouds? And is the distribution of clouds, thermal and nonthermal radio continuum, and infrared sources consistent with stochastic self-propagating star formation as suggested by Gerola et al. $(1980)$ ?

At present, only the star burst phase itself is beginning to be investigated. CO emission in NGC4214 and probably also in DDO 126 was detected with the 30-m IRAM telescope. More important, molecular clouds in NGC3077 and IC10 (see Becker et al. 1988, 1989) could be mapped. The cloud in NGC3077, located on the line of sight toward the central region of this galaxy, has a mass in excess of $10^{7} \mathrm{M}_{0}$ and an FWHP size of 280 pc. Toward the Magellanic irregular 1C10, two clouds are found to be associated with the central bar: one with a size of $180 \mathrm{~d}_{\mathrm{mpc}} \mathrm{pc}$, the other with a size of $\left\langle 40 d_{M p c} p c(d=1-3 M p c)\right.$. Both are associated with $\mathrm{H}_{2} \mathrm{O}$ masers (see Henkel et al. 1986). Also Ohta et al. (1988) reported the detection of clouds in the extended HI envelope of IC10. These observations have been confirmed. More detailed maps reveal in at least one of these 
sources that there are several extended $(>100 \mathrm{pc})$ molecular complexes.

While both NGC3077 and IC10 may, for a variety of reasons, not be "normal" dwarf irregulars, we nevertheless can draw the following very preliminary conclusions:

(1) There are indeed co rich dwarf galaxies.

(2) The detected GMC's are associated with regions of enhanced HI column density $\left(\geq 10^{2 x} \mathrm{~cm}^{-2}\right)$, even in the extended HI shell of IC10.

(3) The detected GMC's are associated with HI "superclouds" (see Elmegreen and Elmegreeen 1987) with typical masses of $10^{7} \mathrm{M}_{0}$, whose existence (as that of the GMC's) appears not to depend on density waves, speed of rotation, degree of shear or metallicity.

(4) The GMC's in the central regions of their parent galaxies are associated with IRAS point sources. This is, however, not the case for the clouds in the HI shell of ICIO.

(5) The GMC's can be larger than those observed in the galactic disk, in spite of the small size of the parent galaxies and the relatively small number of large clouds. Such very large clouds are also seen toward the 30 Dor cloud in the LMC (Cohen et al. 1988), and toward the lenticular galaxy NGC404 (Wiklind and Henkel 1989). All these galaxies have one property in common: massive star formation is not periodically triggered by density waves. Hence more time might be available to build up extended clouds before they are dispersed by newly formed massive stellar objects.

For the future, we expect detailed studies from a large number of dwarf irregulars, located both in the northern and in southern hemisphere, where NGC55 was recently detected with the 15-m SEST telescope (Heithausen, private communication). In addition, investigating the extended HI envelopes of both dwarf and giant irregulars will provide important clues with respect to the unknown origin of this material. Assuming that the CO clouds are in virial equilibrium, a determination of the $\mathrm{I}_{\mathrm{CO}_{0}}-\mathrm{N}\left(\mathrm{H}_{2}\right)$ conversion ratio will provide a first estimate of the metallicity, which can then be compared with theoretical predictions, as e.g. with the models of Dekel and Silk (1986) and Silk et al. (1987).

\section{References}

Becker, R., Appenzeller, I., Wiklind, T., Wouterloot, J.G.A., Henkel, C., Wilson, T.L., Diamond, P.: 1988, Astron. Ges. Abstract Ser. 1, 42 Becker, R., Schilke, P., Henkel, C.: 1989, Astron. Astrophys., in press Cohen, R.S. et al.: 1988, Astrophys. J. 331, L95

Dekel, A., Silk, J.: 1986, Astrophys. J. 303, 39

Elmegreen, B.G., Elmegreen, D.M.: 1987, Astrophys. J. 320, 182

Gallagher, J.S., Hunter, D.A.: 1984, Ann. Rev. Astron. Astrophys. 22, 37 Gerola, H., Seiden, P.E., Schulman, L.S.: 1980, Astrophys. J. 242, 517 Henkel, C., Wouterloot, J.G.A., Bally, J.: 1986, Astron. Astrophys. 155, 193 Ohta, K., Sasaki, M., Saito, M.: 1988, Pub. Astron. Soc. Japan, in press Silk, J., Wyse, R.F.G., Shields, G.A.: 1987, Astrophys. J. 322, L59

Tacconi, L.J., Young, J.S.: 1987, Astrophys. J. 322, 681

Thronson, H.A., Bally, J.: 1986, in "Star Formation in Galaxies", NASA

Conf.Pub. 2466, ed. C.J. Persson, p. 267

Wiklind, T., Henkel, C.: 1989, Astron. Astrophys., submitted Wiklind, T., Rydbeck, G.: 1986, Astron. Astrophys. 164, L22 